\title{
Deformation of film slicks on the water surface. Experiment and model
}

\author{
S. A. Ermakov, O. A. Danilicheva, I. A. Kapustin, G. V. Leschev, A. A. Molkov \\ Institute of Applied Physics RAS, Nizhny Novgorod, 603950, Russia \\ E-mail: stas.ermakov8@gmail.com
}

\begin{abstract}
Marine slicks are considered as imprints of various oceanic/atmospheric processes on the sea surface. Slicks from localized sources of surfactants, e.g. instant oil spills, are determined mostly by physical characteristics of surface films and by small-scale processes like wind waves until the slick scales become comparable with the scales of marine currents, internal waves, sub-mesoscale eddies, etc. At present, our understanding of the slick geometry and its evolution is still insufficient, partly because of the lack of systematic controlled experiments. Previously we reported on the results of some experiments and proposed a simple model of surfactant spills spreading accounting for the surface stresses induced by wind waves. This paper presents new results of field experiments on oil spill evolution and physical mechanisms of this evolution are discussed. The slick shape was studied using a methodology of contouring slicks with a GPS receiver onboard a motorboat. It is obtained that the cross-wind slick size grows quite slowly with time being almost independent on the wind, while the along-wind axis increases rapidly with wind velocity. A mechanism of "oil overflow" is hypothesized to explain the observed effects. A model taking into account the overflow mechanism is developed and is found to be consistent with observations.
\end{abstract}

DOI: $10.21046 / 2070-7401-2020-17-6-97-102$

\section{Introduction}

Evolution of film slicks on the sea surface, including the transformation of slick shape and slick drift is a very important problem, particularly in application to oil spill remote sensing and forecasting of pollution movement in the ocean and coastal areas. The geometry of film slicks, which are clearly seen in radar and optical imagery of the sea surface at low-to-moderate wind conditions, is determined by a number of factors, among them are physical properties of surface films characterized by the surface tension, viscosity, elasticity, and environmental processes - wind velocity, surface waves, currents, etc. Note that our understanding of the dynamics of film spreading is still poor and even some simple questions remained unanswered. One of these questions is 'why do instant oil spills stretch along (or nearly along) the wind with time. One should remind that the spreading of surfactant films in the surface tension regime is determined by the film pressure (the difference between the surface tension of clean water surface and film-covered surface) and by viscous friction. For an unbounded calm water surface, the film spreading is axially symmetric and is described by the Fay model [1]. However, it is well known that an initial round film spot becomes elongated in the wind direction (see, an example in figure 1).

Recently a physical mechanism of slick deformation due to the action of surface stresses induced by wind waves was discussed and a simple physical model was developed in [2-5]. The model was focused on the effect of asymmetric spreading of films due to surface stresses, induced by wind waves. More precisely, the role of the cross-wind components of the induced wave stresses was analyzed and 
the effects of the reduction of spreading speed or even slick compression in the cross-wind direction were revealed. The model has been found consistent, at least qualitatively, with experiment. In this paper some results of new extended experiments are presented and physical mechanisms and a model are proposed to describe peculiarities of the slick shape not only in the cross-wind direction but also of the initial stage of slick evolution along the wind.

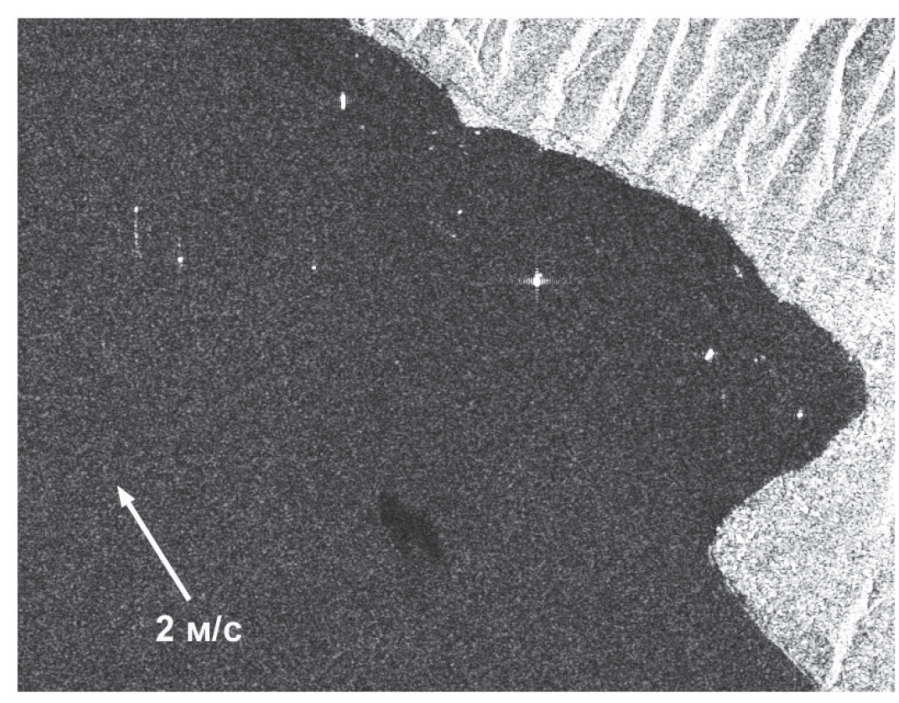

Figure 1. ASAR Envisat imagery of an oil slick. The north-eastern part of the Black Sea (from [2]).

\section{Experiment}

Experiments on film spreading were carried out on the Gorky Water Reservoir in the summer of 2018-2020. Small amounts (about $200 \mathrm{~g}$ ) of oleic acid (OLE) were instantly poured on the water surface, and the slicks were clearly visible because of the strong suppression of short wind waves. The temporal evolution of slick geometry was investigated using a methodology of contouring of the slicks with a motorboat with a GPS receiver onboard. The accuracy of this methodology was confirmed in special although not numerous experiments using aerial photography of oil slicks from UAV nearly simultaneous with the slick contouring. An advantage of aerial photography is that the procedure is non-invasive. However, the capabilities of the photography are limited by small operation times of the UAV (about $15 \mathrm{~min}$ ). Therefore, aerial photography was used mostly to verify the measurements performed by the contouring methodology. The accuracy of the contouring methodology is determined by a) the errors of GPS measurements of coordinates which in fact are no more than 5 meters, b) the finite time of a contouring cycle, when a slick was deforming during the cycle, c) some influence of hydrodynamic disturbances due to the boat on slick spreading. It was shown, however, that both methodologies were consistent with each other, at least within the accuracy of the experiment (see, below). The slick evolution was analyzed at time durations up to about 2 hours, the duration of observations depended strongly on meteorological conditions; at wind velocities larger than $7-8 \mathrm{~m} / \mathrm{s}$ the slicks usually were destroyed and disappeared after about one hour or less. Physical characteristics of OLE films - the elasticity/surface tension coefficient have been studied before the experiments in the laboratory using a parametric wave method [6]. The OLE films over spreading times about 0.5-1 hour could be characterized as oversaturated, i.e. as monomolecular films with microscopic drops of finite thickness. The elasticity and the surface tension of such heterogeneous films can be roughly considered as constants which for OLE-films are both about $30 \mathrm{mN} / \mathrm{m}$ (see [6]).

\section{Results}

An example of slick boundaries obtained using the contouring methodology is given in figure 2 at different times of spreading. It is seen that the slick shape is strongly asymmetric - the slick is stretched along the wind, and the shape is characterized by the occurrence of a "downwind head" and an "upwind tail", these features, however, will not be discussed here.

Results of data processing of the experiments are presented in figure 3 illustrating long/short slick axes for several OLE-slicks as functions of spreading time at different wind velocities. 

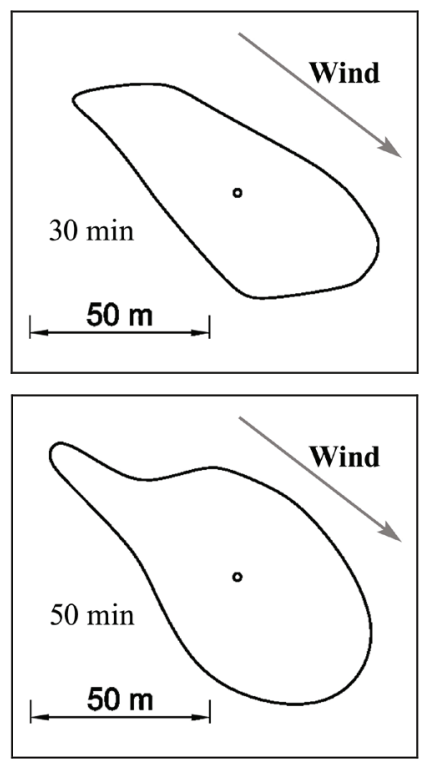

Figure 2. The boundaries of an instant spill of oleic acid in experiments on the Gorky Water Reservoir at different times of film spreading.

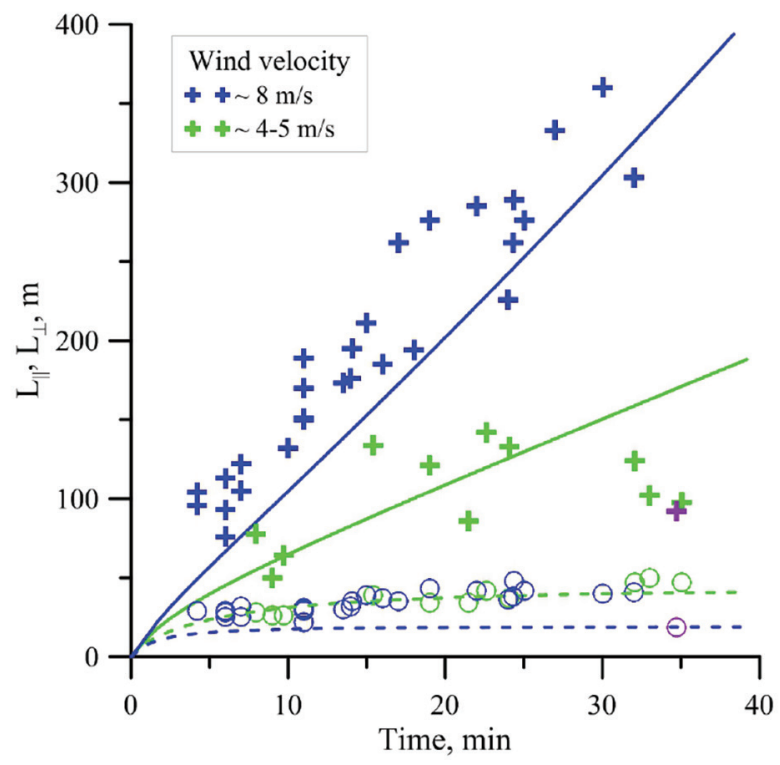

Figure 3. Along-wind (crosses) and cross wind (circles) slick axes vs. spreading time. Theoretical dependencies of along (solid lines) and cross-wind (dashed) slick axes. Blue - wind $8 \mathrm{~m} / \mathrm{s}$, green $-5 \mathrm{~m} / \mathrm{s}$, purple symbols - aerial photography.

It is seen that the along-wind axis was increasing with time faster than the cross-wind axis (cf. [2-5]). A new remarkable fact is that the along-wind axis at an initial stage of slick spreading depends dramatically on wind velocity and the axis growth rate rapidly increases with the wind. On the contrary, the cross-wind axis grows slower with time and weakly depends on wind velocity. At the later stages of film spreading the along-wind axis achieves a maximum and then decreases, tending to the values of the cross-wind axis (cf. [4]), this behavior will be discussed elsewhere. One should note that the initial growth of along-wind axis and its reduction at the later stages was observed at comparably low wind velocities, less than $4-5 \mathrm{~m} / \mathrm{s}$; at stronger wind velocities, e.g., $8 \mathrm{~m} / \mathrm{s}$, the stage of compression was not detected because of slick fragmentation [7].

\section{Theoretical analysis and discussion}

Previously we have reported on a model [4, 5] extending a conventional Fay's theory [1]. The latter is based on the balance between the viscous and surface tension forces acting on a surfactant film on the water surface and predicts axially symmetric spreading of a spill which radius grows with time as $R \sim t^{3 / 4}$. Our approach to the problem of oil spill temporal evolution takes into account mean stresses induced due to nonlinearity by surface waves. The induced stresses act oppositely to the surface tension forces thus resulting in asymmetry of a slick, so that the cross-wind slick axis becomes smaller than the along-wind slick scale. A diagram illustrating different forces acting on a film is shown in figure 4 .

The balance between surface tension, viscous stresses in the surface boundary layer and mean stresses induced by surface waves can be written as following

$$
\Delta \sigma-\alpha \frac{\mu \Delta l_{i}}{t \sqrt{v t}} \Delta l_{i}-\beta \overline{F_{i n d}^{i}} \approx 0
$$

where $\Delta \sigma$ is the film pressure (a difference between the surface tension coefficients for the background water surface and for film covered surface), $\alpha$ - and $\beta$-empirical coefficients, $\Delta l_{i}$ - increments of a slick axes in the cross- or along-wind directions ( $i=\perp$, or $\|$, respectively).

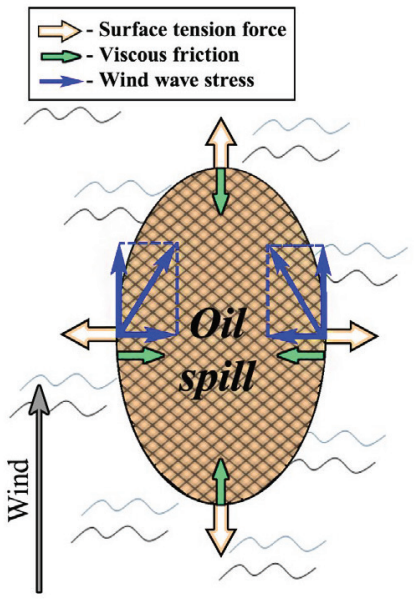

Figure 4. Surface stresses acting on an oil. 
The first, second and third terms in (1) denote surface tension, viscous and induced surface wave forces, respectively. For simplicity we consider further a rectangular slick.

\subsection{Cross-wind axis}

The force due to induced stress acting on a small element $\Delta l_{\perp}$ with a unit length along the slick boundary can be written as (cf. [1])

$$
\bar{F}_{\text {ind }}^{\perp}=\iint \frac{T_{0}}{2 x}\left(1-\mathrm{e}^{-2 x \Delta l_{\perp}}\right) F(k, \varphi) k \mathrm{~d} k \sin \varphi \mathrm{d} \varphi \approx \Delta l_{\perp} \cdot 1 \cdot \tau_{\perp}^{0},
$$

where $\tau_{\perp}^{0}=\iint T_{0} F(k, \varphi) k \mathrm{~d} k \sin \varphi \mathrm{d} \varphi$ denotes a cross-wind component of the wave induced surface stress, $F(k, \varphi)$ the directional wind wave spectrum, $\varphi$ an angle between the surface wave vector and the wind velocity, and $T_{0}$ following to [8] is

$$
T_{0}=\frac{\mu \omega k_{0}}{\delta} \cdot \frac{\beta_{0}^{2}}{|\beta-1+i|^{2}}\left[\frac{1}{\beta_{0}^{2}}-2\left(\frac{x}{k_{0}}+k_{0} \delta\right) \frac{1}{\beta_{0}}+4 k_{0} \delta\right] .
$$

In (3) $\beta=\frac{2 \mu \omega}{E k^{2} \delta}, k=k_{0}+i x, \omega$ and $k_{0}$ are the surface wave frequency and wave number, respectively, $x$ is the wave damping coefficient, $\delta=(2 \mu / \omega \rho)^{1 / 2}$ the viscous boundary layer thickness, $\mu$ the dynamic viscosity of water, $\rho$ the water density, $E$ the film elasticity. The term $4 k_{0} \delta$ in (3) describes the stress component for a clean water surface (at $E=0$ ), it comprises the Stokes drift and the drift caused by the viscous wave damping. The other terms in the r.h.s. of (3) describe the stress at the surface covered with a surfactant film.

\subsection{Along-wind axis}

To describe the temporal evolution of an along-wind slick axis we consider separately the dynamics of upwind, downwind boundaries and the side parts of a slick hypothesizing an "overflow mechanism" of slick spreading. Qualitatively the proposed overflow mechanism can be explained as follows. When calculating along-wind slick scales we took into account only induced stresses acting at the upwind part and side parts of a slick. For the down-wind slick side the induced stresses are negligible since the intensity of wind waves in slick is usually one order of magnitude smaller than outside slick. As a result, the downwind boundary of a slick is supposed to spread according to Fay's model.

The upwind slick boundary dynamics is determined by the action of surface tension, the induced stresses and friction forces and can be described similar to the evolution of cross-wind sides of slicks. The force due to induced stress acting on a small element of $\left(\Delta l_{\|}\right)_{u p}$ in length and a unit width along the upwind slick boundary (up) by analogy with (2) can be written as

$$
\left(\bar{F}_{\text {ind }}^{\|}\right)_{\text {up }} \approx\left(\Delta l_{\|}\right)_{\mathrm{up}} \cdot 1 \cdot\left(\tau_{\|}^{0}\right)_{u p},
$$

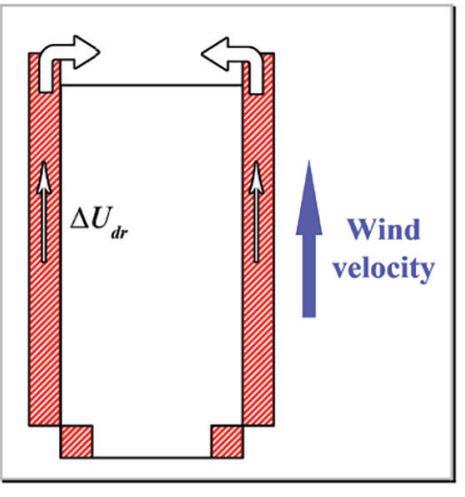

Figure 5. Schematic explanation of the "overflow" effect due to induced wave stresses. where $\tau_{\|}^{0}=\iint T_{0} F(k, \varphi) k \mathrm{~d} k \cos \varphi \mathrm{d} \varphi,\left(\Delta l_{\|}\right)_{u p}$ increment of an upwind slick axis in the along-wind directions.

The side bands of a slick parallel to the wind direction drift along the wind faster than the main body of the slick because of additional wave induced forces. The width of the side bands is of the order of the integral damping length $1 / x_{e f}$ of short wind waves mostly affected by film (typical wavelength range of about $1.5-30 \mathrm{~cm}$ ). The side bands overtake the main slick body and "turn around" to the central axis of the slick under the action of the cross wind components of induced stresses and fill in the area in front of the downwind boundary. In figure 5 we depicted the side bands which are affected by the along-wind components of the wave induced stresses.

The side bands of a slick parallel to the wind direction drift along the wind faster than the main body of the slick because of additional 
wave induced forces. The width of the side bands is of the order of the integral damping length $1 / x_{e f}$ of short wind waves mostly affected by film (typical wavelength range of about $1.5-30 \mathrm{~cm}$ ). The side bands overtake the main slick body and "turn around" to the central axis of the slick under the action of the cross wind components of induced stresses and fill in the area in front of the downwind boundary. In figure 5 we depicted the side bands which are affected by the along-wind components of the wave induced stresses.

The additional drift velocity $\Delta U_{d r}$ of the side bands (sb) can be estimated when balancing the wave induced force $\left(F_{i n d}^{\|}\right)_{s p}$ and the friction force, the latter can be described in the frame of the boundary layer theory for a thin plate as

$$
\beta\left(F_{\text {ind }}^{\|}\right)_{s p} L_{\|}^{0} \approx 0.664 \alpha \sqrt{v \rho \Delta U_{d r}^{3} L_{\|}^{0}} \frac{1}{x_{e f}}
$$

where $\left(F_{\text {ind }}^{\|}\right)_{s p} \approx\left(\varkappa_{e f}\right)^{-1} \cdot 1 \cdot\left(\tau_{\|}^{0}\right)_{s p}$, (one should notice that $\left(\tau_{\|}^{0}\right)_{s p}$ differs from $\left(\tau_{\|}^{0}\right)_{u p}$ because of different integration angular limits when integrating wind wave spectrum in (2)), $L_{\|}^{0}-$ along-wind scale without the overflow effect.

The velocity of the downwind edge $\Delta U_{d r}^{\text {down }}$ due to the overflow effect can be estimated from the balance of the surface-active material as

$$
\Delta U_{d r}^{d o w n} L_{\perp} \approx 2 \Delta U_{d r} \frac{1}{\varkappa_{e f}} .
$$

The downwind slick boundary movement is determined by both spreading due to surface tension and by the overflow effect. So, the coordinate $X_{d}$ of the downwind boundary is

$$
X_{d}=X_{0}(t)+\int \Delta U_{d r}^{d o w n} \mathrm{~d} t .
$$

Where the first term in r.h.s. of (7) is determined by Fay's model and grows as $t^{3 / 4}$.

The along-wind slick axis can finally be obtained when calculating the coordinates of the downwind $(d)$ and upwind (up) slick boundaries (see, also [5]),

$$
\begin{aligned}
L_{\|}(t) \propto X_{d}-X_{u p} & \approx \text { const }+\sqrt{\frac{\Delta \sigma \sqrt{v}}{\alpha \mu}} t^{3 / 4}+\int \Delta U_{d r}^{d o w n} \mathrm{~d} t+ \\
& +\frac{\sqrt{v}}{2 \alpha \mu}\left(\sqrt{\left(\beta\left(\tau_{\|}^{0}\right)_{u p}\right)^{2} t^{3}+4 \Delta \sigma \cdot \alpha \frac{\mu}{\sqrt{v}} t^{3 / 2}}-\beta\left(\tau_{\|}^{0}\right)_{u p} t^{3 / 2}\right) .
\end{aligned}
$$

Theoretical dependencies of along- and cross-wind slick scales on spreading time are shown in figure 3 at wind velocities of 5 and $8 \mathrm{~m} / \mathrm{s}$. The empirical coefficients in (1) were chosen as $\alpha=0.7$ and $\beta=0.47$. The curves in figure 3 were plotted assuming the elasticity of $20 \mathrm{mN} / \mathrm{m}$ and film pressure of $\Delta \sigma=40 \mathrm{mN} / \mathrm{m}$, these values are typical for OLE films at high enough concentrations of about $2-3 \mathrm{mg} \cdot \mathrm{m}^{-2}$ and larger.

For theoretical modeling of the slick evolution we utilized the wind wave spectrum in the form proposed by Elfouhaily et al. in [9]. The angular wave distribution function in the wind wave spectrum was assumed as $(\cos \varphi)^{2}$. Integration in (2) was performed in a wave number range from about 0.2 to $4.5 \mathrm{rad} / \mathrm{cm}$, (wavelengths ranged from 1.5 to $30 \mathrm{~cm}$ ), i.e. over the spectrum of short wind waves which are the most strongly affected by film. When calculating the slick axis in a cross-wind direction we considered symmetric induced stresses acting toward the slick.

\section{Conclusions}

Field experiments with oil slicks were carried out using oleic acid instantly spilled from a motorboat. The slick shape was studied using a methodology of contouring slicks with the boat moving along a slick boundary with a GPS receiver onboard. It was obtained that the slick cross-wind size grows quite slowly with time being weakly dependent on wind velocity. The along-wind slick size increases with wind velocity and grows faster with time compared to low wind conditions. The temporal growth rate 
of the cross-wind axis is smaller than that for the along-wind axis and weakly depends on wind velocity, the dependence of the cross-wind axis can be roughly approximated as $t^{1 / 2}$. A model describing the slick evolution is based on the action of surface stresses induced by wind waves. To explain the obtained dependencies of the along-wind slick size on wind speed a mechanism of oil overflow is hypothesized. This mechanism describes the movement of surfactants from lateral and upwind slick sides to its downwind edge under the action of along-wind components of the wave induced stresses.

\section{Acknowledgements}

This research was funded by the Russian Science Foundation (Project No. 18-17-00224).

\section{References}

[1] Fay J.A., The Spread of Oil Slicks on a Calm Sea, Oil on the Sea, D. P. Houlr (ed.), New York: Springer, 1969, pp. 53-63.

[2] Ermakov S. A., Lavrova O. Yu., Kapustin I. A., Makarov E. V., Sergievskaya I. A., Investigation of geometry of film slicks on the sea surface from satellite radar observations, Sovremennye problemy distantsionnogo zondirovaniya Zemli iz kosmosa, 2016, Vol. 13(3), pp. 97-105.

[3] Ermakov S., Kapustin I., Sergievskaya I., Da Silva J., Spreading of oil films on the sea surface: radar/optical observations and physical mechanisms, Remote Sensing of the Ocean, Sea Ice, Coastal Waters, and Large Water Regions, Proc. SPIE, 2015, Vol. 9638, 963807, 7 p., DOI: 10.1117/12.2195004 963807.

[4] Ermakov S., Kapustin I., Molkov A., Leshev G., Danilicheva O., Sergievskaya I., Remote sensing of evolution of oil spills on the water surface, Remote Sensing of the Ocean, Sea Ice, Coastal Waters, and Large Water Regions, Proc. SPIE, 2018, Vol. 10784, 107840L, 6 p., DOI: 10.1117/12.2325745.

[5] Ermakov S., Danilicheva O., Kapustin I., Molkov A., Drift and shape of oil slicks on the water surface, Remote Sensing of the Ocean, Sea Ice, Coastal Waters, and Large Water Regions, Proc. SPIE, 2019, V. 11150, 111500J, 7 p., DOI: 10.1117/12.2533203.

[6] Ermakov S.A., Kijashko S.V., Laboratory study of the damping of parametric ripples due to surfactant films, Marine surface films, M. Gade, H. Hühnerfuss, G. Korenowski (eds.), Berlin: Springer, 2006, pp. 113-128.

[7] Danilicheva O.A., Leshev G.V., Molkov A.A., Ermakov S.A., On Peculiarities of Spreading of SurfaceActive Substances on Water Under Condition of Strong Wind Wave Breaking, $3^{\text {rd }}$ All-Russian Scientific Conference on the Problems of Ecology of Volga Basin "Volga-2018”: Proc., Nizhny Novgorod, 2018, Vol. 1, $4 \mathrm{p}$.

[8] Foss M., Manifestation of momentum transfer in case of ocean surface waves being damped by an elastic film or a viscous layer, Dissertation Dr. Sci. Norway, University of Tromso, 2000, 128 p.

[9] Elfouhaily T., Chapron B., Katsaros K., Vandemark D., A unified directional spectrum for long and short wind-driven waves, J. Geophysical Research, 1997, Vol. 102(C7), pp. 15781-15796. 